\title{
Mejora de la independencia de las actividades básicas de la vida diaria al implementar fisioterapia manual, neurológica y reeducación de la marcha en paciente geriátrico con diagnostico de enfermedad articular degenerativa y enfermedad de Parkinson. Reporte de un caso clínico
}

Improvement of the Independence of the Daily Basic Life Activities by Implementing Manual Physiotherapy, Neurological and Reeducation of Walk in a Geriatric Patient with Diagnosis of Degenerative Joint and Parkinson's Disease. Clinical Case Report.

\section{Bustamante-Mar $E^{a}$,Porta-Lezama $M^{b}$, Bustamante-Mar $E^{b}$}

\begin{abstract}
:
We present the case of an 80-year-old female patient with non-pathological personal and family history; pathological personal history: attended with trigeminal neuralgia 30 years ago, right ankle fracture 20 years ago, right hip arthroplasty two years ago, recently appeared Parkinson's disease; medicated with memantine, angiotaxil, levodopa and carbidopa referred to the rehabilitation service for degenerative joint disease, polyarthralgia that limits bimanual activities, standing, walking for activities of daily living (BADL) being dependent for bathing and dressing; she received rehabilitation for 4 months with significant improvement to perform activities of daily living in an independent way, included in these bath and dress that were limited.
\end{abstract}

Keywords:

Aging, arthritis, Parkinson, geriatric physiotherapy

\section{Resumen:}

Se presenta caso de paciente femenina de 80 años de edad con los antecedentes heredofamiliares y personales no patológicos sin importancia; antecedentes personales patológicos: cursó con neuralgia del trigémino hace 30 años, fractura de tobillo derecho hace 20 años, artroplastia de cadera derecha hace dos años, enfermedad de Parkinson de reciente aparición; medicada con memantina, angiotaxil, levodopa y carbidopa referida al servicio de rehabilitación por enfermedad articular degenerativa, poliartralgias que le limita las actividades bimanuales, la bipedestación, la marcha para las actividades de la vida diaria (ABVD) siendo dependiente para el baño y vestido; recibió rehabilitación por 4 meses con mejoría relevante para realizar actividades de la vida diaria de forma independiente, incluidas en estas baño y vestido que eran limitantes.

\section{Palabras Clave:}

Envejecimiento, artrosis, Parkinson, fisioterapia geriátrica 


\section{Introducción}

En este trabajo se presenta el caso de una paciente femenina, adulta mayor, con enfermedades neurológicas y traumáticas que es referida a rehabilitación. Posterior a la fisioterapia neurológica que recibió, se observó la mejora de su capacidad para realizar actividades básicas de la vida diaria.

La fisioterapia neurológica ha tenido un gran impacto en el proceso de rehabilitación de la población adulta mayor con este tipo de enfermedades, al permitir mejorar la independencia y funcionalidad de los individuos mediante el desarrollo de las capacidades humanas como es fuerza, equilibrio, coordinación y disminución de la rigidez, teniendo como resultado la capacidad de desempeñarse en su medio con el mínimo esfuerzo y favoreciendo a satisfacer necesidades básicas como son las actividades básicas de la vida diaria.

Este reporte destaca el alcance de la fisioterapia como estrategia de intervención que permite y aletargar la enfermedad mejorando el control motor de las actividades bimanuales así como de la marcha aletargando el proceso de degeneración.

\section{Descripción del caso}

Se presenta caso de paciente femenina de 80 años de edad con los antecedentes heredofamiliares y personales no patológicos sin importancia; antecedentes personales patológicos: cursó con neuralgia del trigémino hace 30 años, fractura de tobillo derecho hace 20 años, artroplastia de cadera derecha hace dos años, enfermedad de Parkinson de reciente aparición; medicada con memantina, angiotaxil, levodopa y carbidopa referida al servicio de rehabilitación por enfermedad articular degenerativa, poliartralgias que le limita las actividades bimanuales, la bipedestación, la marcha para las actividades de la vida diaria (ABVD) siendo dependiente para el baño y vestido; recibió rehabilitación por 4 meses con mejoría relevante para realizar actividades de la vida diaria de forma independiente, incluidas en estas baño y vestido que eran limitantes.

\section{a) Diagnóstico nosológico:}

1. Enfermedad articular degenerativa crónica no transmisible

2. Enfermedad de Parkinson

3. Artroplastia de cadera derecha

b) Diagnóstico etiológico:
Limitación funcional para las actividades básicas de la vida diaria, principalmente bimanuales, movilidad corporal y marcha secundaria/secuela a degeneración del complejo articular de extremidades torácicas y pélvicas, regeneración del sistema extrapiramidal y recambio articular de cadera derecha.

\section{Pronóstico}

Pronóstico funcional bueno para las actividades básicas de la vida diaria bajo permanente supervisión familiar a través de tratamiento fisioterapéutico constante que refuerce la funcionalidad e independencia.

\section{Plan de intervención y resultados}

El plan de tratamiento consistió en 10 sesiones de rehabilitación que se realizaron dos días a la semana con una duración de 30 minutos cada una durante 4 meses, se llevaron a cabo en el área de rehabilitación de la Unidad de Medicina Familiar No 32, cuyos objetivos fueron los siguientes:

\section{Objetivos}

\section{Objetivo general:}

- Aumentar la máxima independencia en la realización de las actividades básicas de la vida diaria.

\section{Objetivos específicos:}

- Aumentar arcos de movimiento de miembros superiores e inferiores.

- Mejorar fuerza en extremidades inferiores.

- Aumentar equilibrio y propiocepción de las extremidades inferiores.

- Mejorar las fases de la marcha mediante el apoyo de andadera con progreso a bastón de un punto.

- Valorar la fatiga muscular del paciente mediante la escala de Borg durante la intervención.

\section{Degenerativo \\ 2. Quirúrgico}

\section{c) Diagnóstico anatomofuncional}


Tabla 1 Plan de intervención

\begin{tabular}{|c|c|c|c|}
\hline Objetivo & Modalidad & $\begin{array}{l}\text { Intensid } \\
\text { ad }\end{array}$ & $\begin{array}{l}\text { Dosificaci } \\
\text { ón }\end{array}$ \\
\hline $\begin{array}{l}\text { Aumentar } \\
\text { arcos de } \\
\text { movimien } \\
\text { to de } \\
\text { extremida } \\
\text { d } \\
\text { superiore } \\
\text { s }\end{array}$ & $\begin{array}{l}\text { Mediante el uso } \\
\text { de cinesiterapia } \\
\text { pasiva } \\
\text { (unilateral y } \\
\text { bilateral). }\end{array}$ & $\begin{array}{l}\text { Borg } \\
3 / 10\end{array}$ & $\begin{array}{l}2 \text { series } \\
\text { de } 10 \text { a } 15 \\
\text { repeticion } \\
\text { es }\end{array}$ \\
\hline $\begin{array}{l}\text { Aumentar } \\
\text { arcos de } \\
\text { movimien } \\
\text { to de } \\
\text { extremida } \\
\text { d inferior }\end{array}$ & $\begin{array}{l}\text { Pararse y } \\
\text { sentarse(privileg } \\
\text { iar la fase } \\
\text { excéntrica). }\end{array}$ & $\begin{array}{l}\text { Borg } \\
3 / 10\end{array}$ & $\begin{array}{l}2 \text { series } \\
\text { de } 10 \text { a } 15 \\
\text { repeticion } \\
\text { es }\end{array}$ \\
\hline $\begin{array}{l}\text { Aumentar } \\
\text { el } \\
\text { equilibrio } \\
\text { de } \\
\text { miembros } \\
\text { pélvicos }\end{array}$ & $\begin{array}{l}\text { Educación de } \\
\text { estrategias ante } \\
\text { perturbaciones } \\
\text { del equilibrio } \\
\text { propiocepción } \\
\text { (cadera,rodilla, } \\
\text { tobillo). } \\
\text { Ejercicios de } \\
\text { Frenkel sobre } \\
\text { extremidades } \\
\text { inferiores. }\end{array}$ & $\begin{array}{l}\text { A } \\
\text { toleranci } \\
\text { a }\end{array}$ & $\begin{array}{l}5 \text { a } 8 \\
\text { minutos } \\
3 \quad \text { series } \\
\text { de } 5 \\
\text { repeticion } \\
\text { es con } \\
\text { duración } \\
\text { de } 10 \\
\text { segundos } \\
\text { con } \\
\text { intervalos } \\
\text { descanso } \\
\text { de } 10 \\
\text { segundos } \\
\text { entre cada } \\
\text { serie }\end{array}$ \\
\hline $\begin{array}{l}\text { Mejorar } \\
\text { equilibrio } \\
\text { estático }\end{array}$ & $\begin{array}{lr}\text { Marcha } & \text { con } \\
\text { andadera con } \\
\text { progresión a } \\
\text { bastón de un } \\
\text { punto y } \\
\text { posteriormente } \\
\text { a marcha } \\
\text { independiente. }\end{array}$ & $\begin{array}{l}\text { A } \\
\text { toleranci } \\
\text { a }\end{array}$ & 5 minutos \\
\hline
\end{tabular}

En este caso, se encontró que al implementar fisioterapia neurológica, manual se pudo obtener una ganancia en las actividades de baño y vestido, pasando de ser dependiente que necesita ayuda de un tercero a totalmente independiente, así como una mejora en la marcha y en las demás actividades como es el uso del WC, movilización, continencia y alimentación; mejorando además arcos de movimientos, mejora en la espasticidad y mejora en las fases de la marcha (Tabla 2).
Tabla 2 Mejora de la independencia de las actividades de la vida diaria mediante la implementación de fisioterapia.

$\begin{aligned} & \text { Actividad antes de la } \\
& \text { intervención }\end{aligned}$
\begin{tabular}{|l|l|l|l|}
\hline Baño & $\begin{array}{l}\text { Actividad después de la } \\
\text { intervención } \\
\text { asistencia }\end{array}$ & Baño & $\begin{array}{l}\text { Independie } \\
\text { nte }\end{array}$ \\
\hline Vestido & $\begin{array}{l}\text { Requiere } \\
\text { asistencia }\end{array}$ & Vestido & $\begin{array}{l}\text { Independie } \\
\text { nte }\end{array}$ \\
\hline $\begin{array}{l}\text { Continenci } \\
\text { a }\end{array}$ & $\begin{array}{l}\text { Independie } \\
\text { nte }\end{array}$ & $\begin{array}{l}\text { Continenci } \\
\text { a }\end{array}$ & $\begin{array}{l}\text { Independie } \\
\text { nte }\end{array}$ \\
\hline $\begin{array}{l}\text { Uso de } \\
\text { W.C. }\end{array}$ & $\begin{array}{l}\text { Dependient } \\
\text { e }\end{array}$ & $\begin{array}{l}\text { Uso de } \\
\text { W.C. }\end{array}$ & $\begin{array}{l}\text { Independie } \\
\text { nte }\end{array}$ \\
\hline Movilidad & $\begin{array}{l}\text { Dependient } \\
\text { e }\end{array}$ & Movilidad & $\begin{array}{l}\text { Independie } \\
\text { nte }\end{array}$ \\
\hline $\begin{array}{l}\text { Alimentaci } \\
\text { ón }\end{array}$ & $\begin{array}{l}\text { Dependient } \\
\text { e }\end{array}$ & $\begin{array}{l}\text { Alimentaci } \\
\text { ón }\end{array}$ & $\begin{array}{l}\text { independie } \\
\text { nte }\end{array}$ \\
\hline
\end{tabular}

\section{Discusión}

El programa de rehabilitación está destinado a mejorar de forma progresiva la fuerza, el aumento de las actividades de la vida diaria que no se podían realizar así como la mejora en la marcha, ya que las cargas físicas progresivas y programadas de forma personalizada ejercen mejor funcionalidad y evolución en el entrenamiento de fuerza, equilibrio y de la marcha, evitando la sobrecarga de esfuerzo al realizar entrenamientos con muchas repeticiones, así como evitar cualquier tipo de lesión asociada al sobreentrenamiento.

En función de las valoraciones médicas se realizaron intervenciones en fisioterapia neurológica, manual y en rehabilitación de la marcha. En la tabla 1 se presentan los tipos de ejercicios tanto neurológicos, de propiocepción, equilibrio así como movilizaciones que permitieron llegar al cumplimiento de los objetivos planteados, dicha rehabilitación se realizó en sesiones de 2 veces por semana durante 4 meses; dichas intervenciones se modificaron mediante progresiones respectivas para favorecer el desarrollo de adaptaciones constantes y controladas que llevaran al paciente a mejorar sus capacidades anatomofuncionales, como lo es resistencia muscular, equilibrio, coordinación, propiocepción, marcha y mejora en ABVD. Como parte de la estrategia terapéutica se valoró mediante la escala de Borg de esfuerzo percibido la cual permite la dosificación de la carga de entrenamiento, apreciar la respuesta del organismo y realizar un control en donde se deberá evaluar el esfuerzo de acuerdo a una escala numérica de 11 niveles; 0 a 2 esfuerzo suave, 3 a 4 esfuerzo moderado, 5 a 6 esfuerzo duro, 7 a 9 esfuerzo muy duro y 10 esfuerzo máximo.

\section{Conclusiones}

La estrategia establecida por el área de rehabilitación para el restablecimiento de las capacidades motoras de extremidades superiores e inferiores y la reeducación de la marcha, permitió restablecer las actividades de baño y vestido como parte de las actividades básicas de la vida 
diaria, sin necesidad de apoyo de un tercero y permitiendo dejar los instrumentos de apoyo como silla de ruedas, andadera y bastón de un punto.

Los adultos mayores que presentan alguna enfermedad neurológica tienen mayor dependencia para realizar actividades que aquellos que no la tienen. Esto puede explicarse si se toma en cuenta que, al ser personas de avanzada edad, los padecimientos neurológicos son generalmente de larga evolución, aunado a la falta de actividad física y a otros componentes de los estilos de vida saludable. Ante este escenario la fisioterapia neurológica juega un papel predominante para incorporar al individuo afectado a la capacidad de realizar sus actividades cotidianas con independencia.

\section{Referencias}

[1] Morales S. [Tesis para especialidad Internet].[Tenerife] Programa de intervención Logopédica en el habla y la voz de afectados con Parkinson. Universidad de la laguna.2016.

[2] Pérez C, Morales CM, Ortiz D, Castellano OM, Suárez YL. [Tesis para especialidad Internet]. [Bogotá] Funcionalidad del miembro superior afectado en personas con secuelas de enfermedad cerebrovascular. Institución Universitaria Fundación Escuela Colombiana De Rehabilitación. 2010.

[3] Viesca TC. Paternalismo médico y consentimiento informado. En: Márquez-Romero R, Rocha- Cacho WV, editors. Consentimiento informado. Fundamentos y problemas de su aplicación práctica. 1st ed. México: UNAM; 2017: 1-22.

[4] Reyes H, Vladisnoca D, García J, Jiménez R, Peña A, Mendoza V. Guía para la evaluación gerontológica integral. Rev Med Inst Mex Seguro Soc. 2009; 47 (3): 291-306.

[5] González R, Cardentey J. Comportamiento de las enfermedades crónicas no transmisibles en adultos mayores. Rev Finlay. 2018;8(2):1-7.

[6] Barba J. México y el reto de las enfermedades crónicas no transmisibles. El laboratorio también juega un papel importante. Rev Latinoam Patol Clin Med Lab. 2018; 65 (1): 4-17.

[7] Espinoza G, López VM, Escobar DA, Conde M, Trejo G, González B. Programa para la rehabilitación funcional del adulto mayor para mejorar la marcha, el equilibrio y la independencia. Rev Med Inst Mex Seguro Soc. 2013;51(5):562.

[8] Cash D. Neurología para fisioterapeutas; Buenos Aires, Argentina: Editorial Médica Panamericana. 2001:568.

[9] Chandler C, Plant R.Parkinson's disease: Studies in psychological and social care. Leicester: BPS Books, 1999:256-269.

[10] Trigas M, Ferreira L, Mejide H.Escalas de valoración funcional en el anciano. New Eng Journal Med:1990;322: 1207-1214. 